\title{
Reestruturação Curricular na Faculdade de Medicina da USP: Integrando Conteúdos e Valorizando Competências
}

\author{
Luiz Fernando Ferraz da Silva*1, Edmund Chada Baracat ${ }^{2}$ e \\ José Otavio Costa Auler Junior ${ }^{3}$
}

'Departamento de Patologia, ${ }^{2}$ Departamento de Obstetrícia e Ginecologia e ${ }^{3}$ Departamento de Cirurgia

Faculdade de Medicina da Universidade de São Paulo

* Autor para correspondência: burns@usp.br

\section{RESUMO}

Buscando o contínuo aprimoramento de seu processo de ensino-aprendizagem, a Faculdade de Medicina da USP desenvolveu, no período de 2011 a 2014, um conjunto amplo de atividades visando à reestruturação curricular do curso médico mediante a adoção de um currículo baseado em competências. Esse processo envolveu: (a) uma etapa preliminar que incluiu avaliação curricular externa e formação de oito grupos de trabalho temáticos com o objetivo de integrar conteúdos correlatos e (b) uma etapa de estruturação na qual foram desenvolvidos os conceitos e estratégias de integração básico-clínica, internato, avaliação, governança e capacitação docente. Discutiu-se amplamente todo o processo com a comunidade por meio de reuniões departamentais, congregações temáticas e reuniões de grupos de trabalho. Por aclamação, a congregação da FMUSP aprovou, em 2014, a nova estrutura, a qual teve sua implementação iniciada para os ingressantes em 2015.

Palavras-chave: Currículo; Medicina; Integração; Currículo Baseado em Competências.

\begin{abstract}
University of São Paulo Medical School (FMUSP) have been continuously seeking to maintain its leadership by making improvements on its teaching/learning process. From 2011 to 2014 we developed a pool of activities aiming a strong reformulation towards a competency based medical curriculum. It was developed as a two step approach. The first (preliminary) step, in which FMUSP's curriculum was independently evaluated by foreign institutions and 8 thematic working groups were carried out aiming the initial integration of related contents. The second (structural development) step, in which the concepts and strategies have been developed regarding the basic-clinical integration, internship rotations, evaluation, governance and faculty development. The process was widely discussed within the community through departmental meetings, thematic open meetings and workshops. The new curriculum was approved by acclamation by the Faculty Senate in 2014 and had its implementation started for freshmen in 2015.
\end{abstract}

Keywords: Curriculum; Medicine; Integration; Competency Based Curriculum.

\section{Introdução}

A Faculdade de Medicina da Universidade de São Paulo (FMUSP) sempre buscou estar na vanguarda do desenvolvimento do ensino médico nacional e sempre teve consciência da importância da adaptação frequente em virtude do surgimento de novas estratégias de ensino-aprendizagem mais consoantes com as novas gerações de alunos e profissionais e com as necessidades formativas da área. Nesse sentido, em 2011, a FMUSP deu início a uma série de atividades e discussões que culminaram na implementação de sua nova es- trutura curricular integrada, baseada em competências para ingressantes no ano de 2015. Neste relato, apresentaremos as seis etapas trilhadas em tal processo e, por fim, alguns pilares conceituais da nova estrutura curricular da unidade.

\section{Atividades preliminares - Etapas 1 e 2}

As atividades preliminares visaram à identificação dos principais problemas e dificuldades da estrutura curricular vigente, além de já buscarem proposições de melhoria que pudessem ser implementadas de imediato, antes da reformulação 
curricular completa. Destacam-se, a seguir, duas ações nesse sentido.

A primeira foi a criação de oito Grupos de Trabalho (GT) de Alinhamento de Conteúdos. Esses grupos apresentaram temáticas integradas por sistemas como, por exemplo: aparelho locomotor, aparelho digestório, aparelho cardiovascular, entre outros. Em cada GT, participaram professores de áreas básicas e clínicas procurando, em reuniões quinzenais e mensais, identificar os conteúdos e alinhá-los de modo a otimizar a abordagem, possibilitar integração clínica precoce e fazer uso racional de repetições ao longo das disciplinas. Dentre os frutos dessa iniciativa podem-se enumerar as seguintes medidas: criação de matrizes de conhecimento; inclusão de discussões práticas de casos em disciplinas básicas como anatomia e fisiologia; disponibilização de material didático entre disciplinas diferentes; alinhamento temporal de conteúdos de forma mais racional. Os referidos grupos atuaram durante o ano de 2011 e 2012, e realizaram-se fóruns semestrais para integração entre os GTs.

Considerando-se ainda que, por mais críticos e observadores que sejam os membros do corpo docente e discente em relação às deficiências, potencialidades e fragilidades do currículo, o olhar externo revela-se sempre capaz de agregar pontos que, de outra maneira, passariam desapercebidos. Dessa maneira, colaborações com instituições do exterior, que incluíram Brown University, University of Toronto e National Board of Medical Examiners, permitiram uma avaliação sistematizada do currículo e estruturas da FMUSP durante os anos de 2012 e 2013. Após visitas a estruturas físicas e didáticas, além da realização de entrevistas com dirigentes, professores e alunos, foram obtidos relatórios específicos de análise SWOT (Forças, Fraquezas, Oportunidades e Ameaças). Tais relatórios foram discutidos em oficinas com professores e alunos na tentativa de construção de uma agenda inicial que abordasse estes problemas e utilizassem as forças e oportunidades observadas.

Combinando a experiência prévia dos Grupos de Trabalho e os relatórios de avaliação, foram planejadas novas oficinas, algumas delas até mesmo com a presença dos colaboradores internacionais, que marcaram o início da concepção e desenho do novo currículo que já pressupunha ser um currículo baseado em competências, com grande integração e contextualização básico-clínica organizada em módulos, avaliação formativa estruturada e uma abordagem moderna e dinâmica, incluindo redução da carga horária presencial, atividades extraclasse e uma base informatizada para estudo e acompanhamento de conteúdos.

\section{Desenvolvimento - Etapas 3 a 6}

Para trabalhar estas ideias iniciais e discutir as possibilidades de mudança e implementação de forma prática e objetiva, a terceira etapa consistiu na criação de cinco GT's a saber: 1) Integração básico-clínica; 2) Internato; 3) Avaliação formativa; 4) Capacitação docente; e 5) Comitê Central que, além de gerenciar os demais, ainda teve a atribuição de definir a nova estrutura de governança curricular.

Os resultados iniciais do trabalho desses grupos, que incluíram professores de diferentes disciplinas e representantes discentes, foram os seguintes:

1 - Integração básico-clínica: mudança da estrutura disciplinar; criação de blocos temáticos baseados em órgãos/sistemas e blocos integrativos de múltiplas competências (Figura 1); período de atividades extraclasse de pelo menos $30 \%$.

2 - Internato: reorganização dos estágios de internato e alinhamento, tendo em vista a abertura de possibilidade de estágios optativos de internato na própria FMUSP ou em outras instituições.

3 - Avaliação: desenvolvimento de avaliação semestral formativa independente da aplicação de provas internacionais (modelo IFOM ${ }^{1}$ - International Foundations of Medicine).

4 - Capacitação docente: montagem de oficinas e atividades que buscavam capacitar os professores em novos conceitos da estrutura curricular como ensino baseado em competências; 


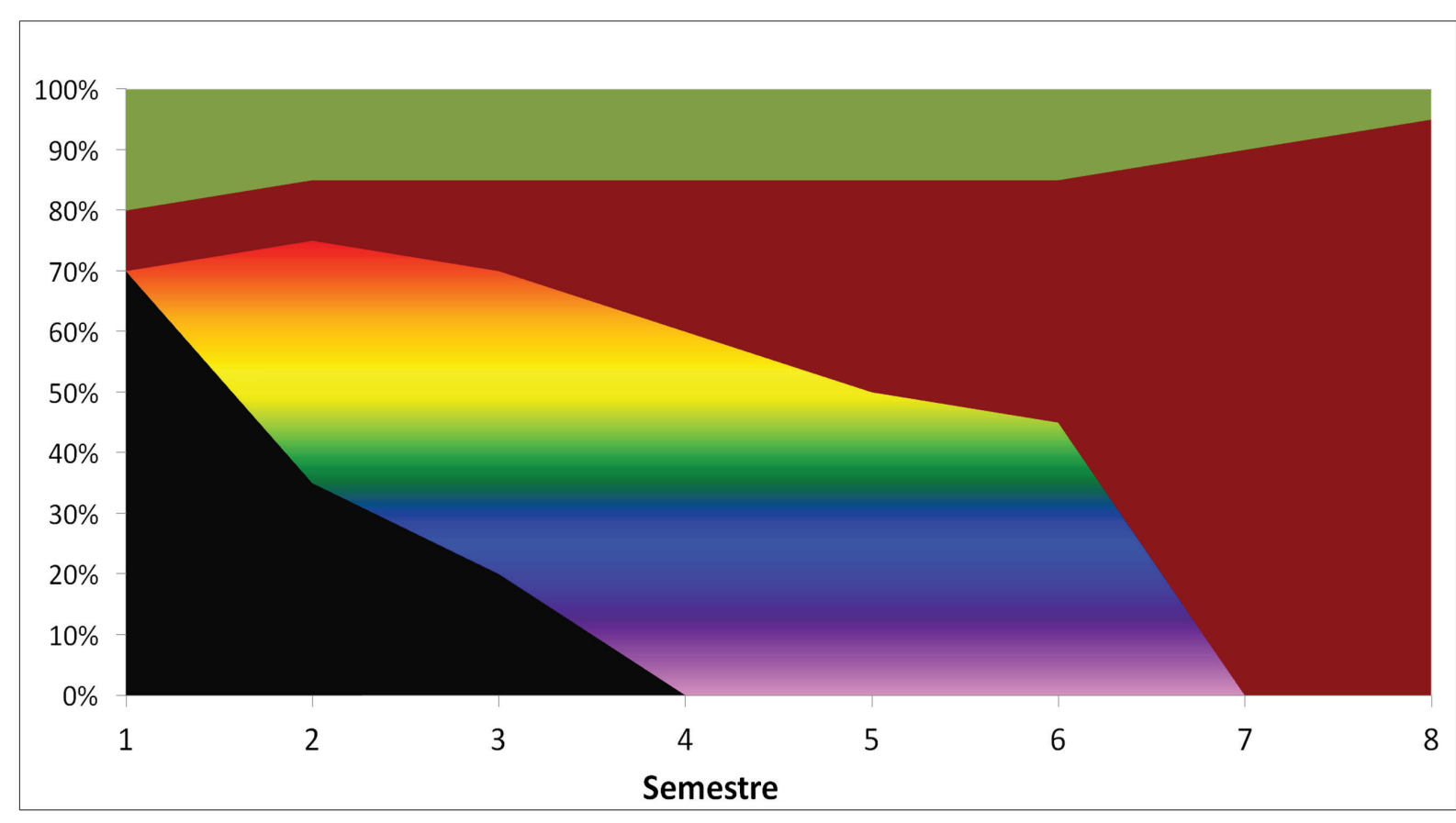

Fundamentos da Medicina = Orgãos e Sistemas

- Integ Competências

Transversais

\section{Fundamentos da Medicina}

UCO - Introdução à Medicina e à Saúde

UC1 - Fundamentos da Ciência Médica

UC2 - Fundamentos Morfofuncionais da Medicina

UC3 - Princípios do Desenvolvimento das Doenças

UC4 - Saúde - Doença - Cuidado

UC29 - Princípios da Investigação Científica em Medicina

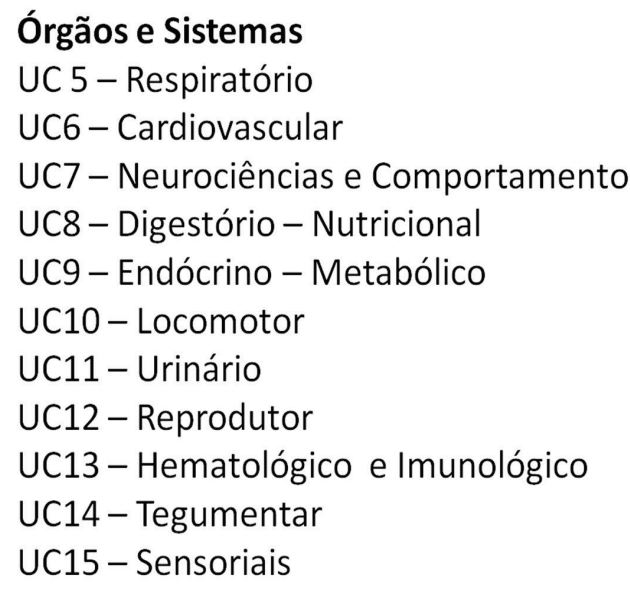

Transversais

UC16 - Ciclos de Vida

UC17 - Oncologia

UC18 - Infecções

UC19 - Terapêutica

UC20 - Reabilitação

\section{Integração de Competências}

UC21 - Discussão Integrada de Casos

UC22 - Prática Ambulatorial

UC23 - GSS Clínicas

UC24 - GSS Cirúrgicas

UC25 - GSS Pediátricas

UC26 - GSS Geriátricas

UC27 - GSS Gineco-Obstétricas

UC28 - GSS Neurocomportamentais

GSS - Grandes Síndromes e Situações

Figura 1 - Mapa curricular das trinta unidades curriculares dos primeiros quatro anos do curso médico da FMUSP divididas de acordo com o grupo estrutural - semestres na abscissa e percentual do componente obrigatório na ordenada.

desenvolvimento e uso de objetivos educacionais e ferramentas tecnológicas de ensino; simulação, estratégias de ensino modelo PBL (Problem-based Learning e Team-based Learning) etc.

5 - Comitê Central: definição da governança (Figura 2), com a sistematização da Comis- são Coordenadora de Curso (CoC) enxuta (oito membros, incluindo professores das unidades envolvidas no curso e um representante discente) e atividade subordinada à Comissão de Graduação (GG - esta com representação departamental), auxiliada por grupos de trabalho permanentes 


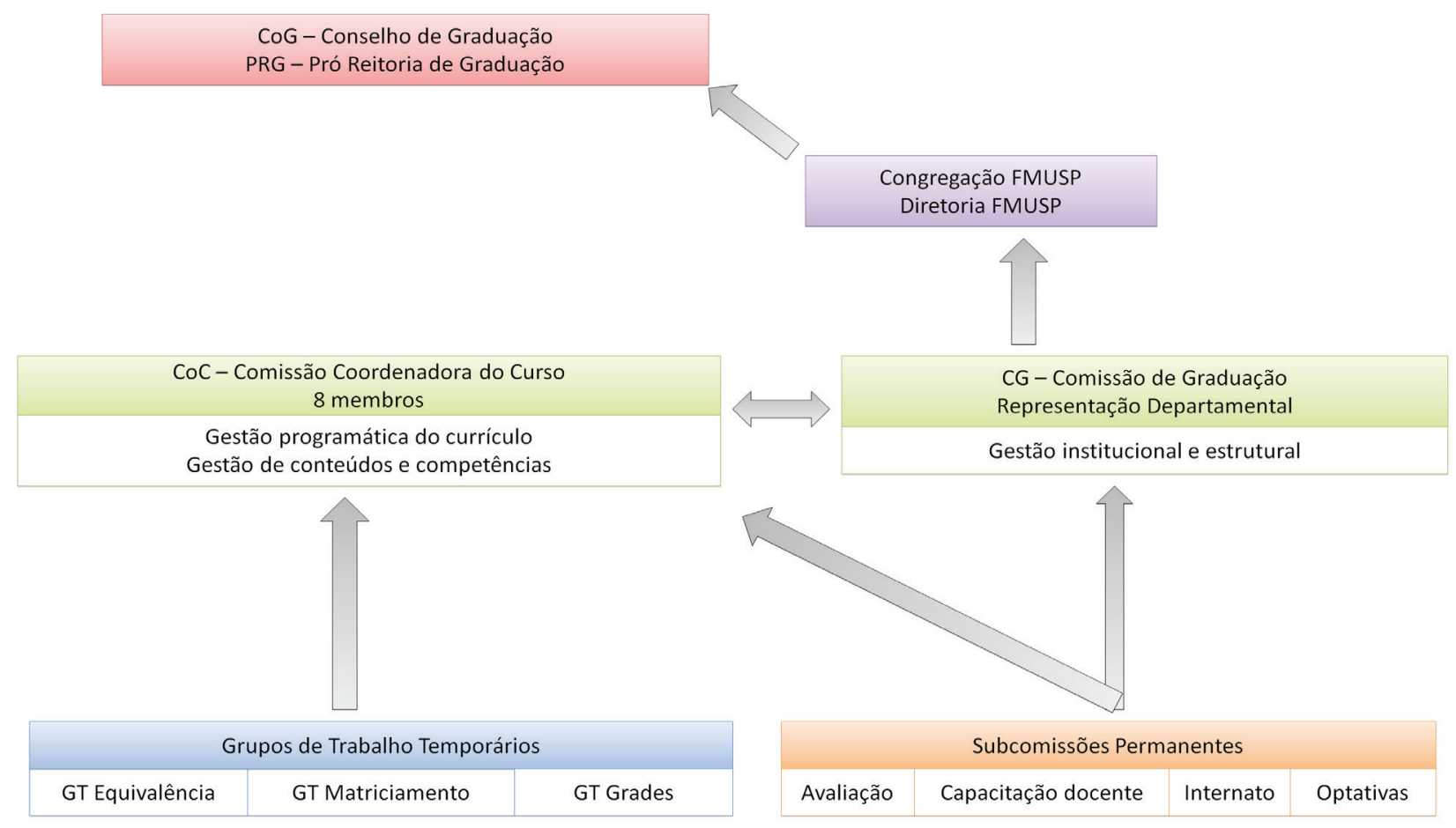

Figura 2 - Modelo de governança curricular FMUSP.

("Capacitação Docente", "Avaliação" e outros que a CoC/CG julgarem necessários).

Os resultados da atuação desses grupos de trabalho levaram à quarta etapa, que envolveu a compilação e integração dos referidos resultados em uma proposta inicial de reformulação curricular, já contendo os principais pilares da reforma curricular (ver a seguir) e que seria submetida então às adaptações e lapidações de toda a comunidade.

Na quinta etapa, a presente proposta foi apresentada e discutida em todos os departamentos da FMUSP, onde foram colhidas sugestões de adaptações e alterações estruturais ou programáticas. Ela também foi apresentada e discutida em duas oficinas organizadas pelo Centro Acadêmico e em uma congregação temática aberta à comunidade.

Com as sugestões obtidas, desenvolveu-se nova rodada de adaptações até ser finalizada a estrutura curricular final, última etapa do processo, que foi submetida à apreciação da Congregação da faculdade em junho de 2014 e aprovada por aclamação para início em 2015. A proposta seguiu então para aprovação das con- gregações das demais unidades envolvidas no curso. Após a aprovação nessas câmaras, foram definidos coordenadores para cada módulo/unidade curricular que permitiram trabalhar, em conjunto com professores das áreas envolvidas e representantes discentes, na estrutura detalhada e programação de cada uma das unidades.

Para facilitar a integração dos envolvidos em tal processo, em agosto de 2014, promoveu-se um encontro geral, envolvendo 147 docentes (FM, HC-FMUSP, HU, ICB, IQe IB) e representantes discentes para o desenvolvimento dos aspectos gerais, objetivos e competências relativas às diferentes unidades curriculares, o que proporcionou mais facilidade e agilidade à discussão de pontos passíveis de integração entre as novas disciplinas.

\section{Pilares da Reestruturação Curricular da FMUSP}

Os principais pilares do novo currículo da FMUSP incluem:

- Currículo baseado em competência, com carga horária de cerca de 7500 horas (redução de 30\% em relação ao currículo anterior), abrindo oportuni- 
dades para o desenvolvimento de atividades extraclasse no âmbito das próprias unidades curriculares (disciplinas) e atividades complementares, como iniciação científica, extensão acadêmica, atividades esportivas, entre outras;

- Abordagem mais dinâmica e integrada, com ênfase para atividades em pequenos grupos e trabalho em equipe, além do uso de ferramentas tecnológicas interativas e plataformas online para disponibilização de material e desenvolvimento de tarefas extraclasse;

- Divisão dos primeiros quatro anos de curso em trinta unidades curriculares organizadas em órgãos/sistemas ou conjuntos de conhecimentos e competências, de modo a facilitar a integração de conteúdos e atividades entre departamentos e institutos, e favorecer a interação clínica e o uso de exemplos práticos aplicados à área médica;

- Reorganização do internato com abertura de espaços para o desenvolvimento de estágios opcionais em áreas específicas de interesse do aluno na FMUSP ou em outras instituições;

- Estruturalmente cada unidade curricular corresponde a uma disciplina interunidades ou disciplina supradepartamental. Considerando-se o conceito de disciplinas departamentais, estas ficaram restritas aos estágios de internato nos dois últimos anos;

- Introdução de uma unidade curricular no início do curso que seria de transição para o aluno entre o Ensino Médio e o Ensino Superior não apenas em termos de conteúdo, mas também nas estratégias de aprendizado e avaliação (busca ativa de conhecimento, estudo individual extraclasse etc.);

- Processo de avaliação formativa permanente com exames semestrais e bianuais independentes para acompanhamento do nível dos alunos e do ensino, com avaliação internacional periódica do programa de Graduação em Medicina pelo International Fundations of Medicine (IFOM);

- Governança curricular que possibilita dinamismo e adaptações rápidas, características fundamentais para um currículo moderno numa sociedade em contínua transformação. Essa estrutura de governança possibilita rediscussão e avaliação contínua da grade, aspecto fundamental para a maturação de um currículo recém-implantado como o nosso.

\section{Desafios e perspectivas}

Apesar do grande apoio de alunos e professores, não se espera unanimidade numa instituição inteligente e diversa como a FMUSP, ao contrário. Os diferentes pontos de vista que dentro do possível foram incorporados na nova estrutura, continuarão a surgir e são fundamentais para estimular o constante aperfeiçoamento da estrutura ora em vigor.

Os momentos iniciais são os mais complexos e difíceis. Numa reformulação tão extensa como a que foi aprovada, todos são imediatamente retirados de sua zona de conforto e chamados a encarar o desafio do novo. Com determinação e cientes da importância dessas alterações, o novo não nos amedronta, ao contrário, estimula-nos a continuar buscando a superação e a excelência, e a liderar um caminho de transformações no ensino médico no Brasil. Afinal, essa é nossa vocação e nossa responsabilidade para com a USP, para com os nossos alunos e para com a sociedade em geral e com nossos pacientes em especial!

\section{Nota}

1. International Fondations of Medicine (IFOM) é um exame preparado pelo National Board of Medical Examiners (NBME) que se mostra similar à prova clínica de habilitação aplicada aos alunos de medicina nos Estados Unidos (USMLE - Step 2).

\section{Referências Bibliográficas}

FRANK, Jason R.; SNELL, Linda; SHERBINO, Jonathan (eds.). CanMeds 2015 - Physician Competency Framework. Ottawa, ON: Royal College of Physicians and Surgeons of Canada, 2015.

UNIVERSIDADE DE SÃO PAULO. Comissão de Graduação da Faculdade de Medicina da USP. Reformulação Curricular da Graduação.

UNIVERSIDADE DE SÃO PAULO. Faculdade de Medicina da USP. Proposta de Reestruturacãa Curricular 2014 -Integrasãa Básico-Clínico / Clínico-Básico - $1^{0}$ ao $4^{\circ}$ Ano.

VIEIRA, Joaquim Edson; SILVA L. F. F.; BARACAT, E. C.; "Medical Education at the University of São Paulo Medical School". Climics, vol. 70, n. 4, 2015, pp. 229-230.

Publicado em 05/07/2016. 
